\title{
Organization Assigning a Unique Protocol Identifer
}

National Cancer Institute

\section{Source}

National Cancer Institute. Organization Assigning a Unique Protocol Identifer. NCI

Thesaurus. Code C64210.

The name of the organization who issued the extension or identifier. 